\title{
When optical networks meet wireless systems: experiments at the boundary
}

\author{
Valerio Frascolla, Intel Deutschland GmbH, Neubiberg, Germany \\ valerio.frascolla@intel.com \\ Carlos Colman-Meixner, Reza Nejabati, Dimitra Simeonidou, University of Bristol, Bristol, UK \\ \{carlos.colmanmeixner, reza.nejabati, dimitra.simeonidou\}@bristol.ac.uk \\ Frank Slyne, Yi Zhang, Luiz A. DaSilva, Marco Ruffini Trinity College, Dublin, Ireland \\ \{fslyne, zhangy8, dasilval, ruffinm\}@tcd.ie

\begin{abstract}
Magnos Martinello, Rafael S. Guimarães, Moises Renato Nunes Ribeiro, Universidade Federal do Espírito Santo, Vitória, Brazil
\end{abstract} \\ magnos@inf.ufes.br, rafael@rafaelguimaraes.net, moises@ele.ufes.br
}

\begin{abstract}
One of the most important announced assets of the forthcoming 5G network is the capability to merge different technologies in an overarching much more effective telecommunication system. At the verge of $5 \mathrm{G}$ deployment, it's therefore key to have the right means to test and pre-develop the integration of two main pillars of $5 \mathrm{G}$, i.e. wireless and optical systems. The target of this paper is to discuss some interesting aspects of performing experiments at the wirelessoptical boundary, focusing on the activities of the EU-Brazil funded research project FUTEBOL.
\end{abstract}

Keywords-Wireless system, Optical network, Testbed, 5G, Experiments, Wireless/optical convergence.

\section{INTRODUCTION}

To serve more than 5.5 billion unique mobile subscribers and 20 billion Internet of Things (IoT) devices connected by 2020 [1], telecommunication networks, leveraging on the $5 \mathrm{G}$ paradigm, will have to smoothly integrate wireless, packet, and optical technologies. Considering the very fast pace of introduction of new features for wireless services users [2], experimentation facilities and testbeds capable of reproducing realistic scenarios become more and more important for both academia and industry, in order to properly design products that can fulfil the forthcoming new $5 \mathrm{G}$ system requirements.

Several testbed facilities have been created through EUfunded collaborative research programmes, like the currently ongoing FP8 (H2020), steered by the Future Internet and Research Experimentation (FIRE) initiative [3]. Leveraging on the achievements of existing testbeds, the EU-Brazil cofunded research project Federated Union of Telecommunication Research Facilities for EU-Brazil Open Laboratory (FUTEBOL) [4] aims to integrate optical, wireless, packets, and cloud domains in an open facility, which can be used for free by experimenters from all over the world. FUTEBOL is a three-year project which started in 2016 and, making use of multiple federated testbeds, already allows the research community to make experimentations towards a convergent optical and wireless network.

This paper focuses on a few key aspects of experiments conducted at the wireless-optical boundary, with the final aim of stressing the increasing importance of wireless-optical integration in future communication networks. The paper is structured as follows: Section II highlights needed enhancements for making wireless-optical integration happen. Section III shows a concrete example of how a network orchestration can be used for optical-wireless integrated experimentations, and Section IV elaborates on remote controlling of a robot using a wireless-optical-cloud infrastructure. Finally, section $\mathrm{V}$ provides the paper conclusion and future work.

\section{ENHANCEMENTS TOWARDS A WIRELESS-OPTICAL CONVERGED FRAMEWORK}

Control Framework (CF) and network orchestration entities are among the most important building blocks needed to realize the optical-wireless convergence in the forthcoming integrated $5 \mathrm{G}$ network. In this section we briefly report some key enhancements that FUTEBOL is currently working on, and which will constitute the basis of the open facility, which will allow the international research community to run experiments from remote.

FUTEBOL enhances existing CFs in several ways [5], among which:

(i) provision heterogeneous resources from existing testbeds through aggregate managers that provide Slicebased Federation Architecture (SFA) interfaces;

(ii) offer a catalogue of services that provides virtual network functions (VNFs), services, and tools that facilitates converged experimentation;

(iii) orchestrate the experiments running in the facility by offering capabilities of inter-testbed live-migration, functional converged network orchestration, and networking and cloud convergence;

(iv) advance network programmability by extending the range of configurable physical parameters that are exposed to the network controllers for a large variety of resources (packet and optical switches, wireless and IoT devices, etc.).

FUTEBOL also extends application-based network orchestration (ABNO) to work as a convergent network orchestrator in the FUTEBOL CF. Typically SDN orchestration for multi-domain networks is designed as monolithic SW which is not flexible enough for meeting heterogeneous networks requirements.

To achieve convergent network programmability, SDN solutions should not work as a "black box". That's why the FUTEBOL ABNO components were constructed in such a way that it is possible to abstract the physical characteristics of the devices, and therefore only few ABNO modules need to be extended to support different network elements. That allows to more effectively orchestrate the end-to-end (E2E) 
control, e.g. from the optical core network testbed at the University of Bristol (UNIB), to the Cloud-RAN based Passive Optical Network (PON) and LTE configurable radio testbed at Trinity College Dublin (TCD), being UNIB and TCD two of the partners of the FUTEBOL project. Specific retrievers and dispatchers were developed for the physical layer parameters of multi-domain networks.

More details on exemplary experiments that can be run by the facility created by FUTEBOL can be found in [6]. In the following sections we introduce two examples.

\section{REMOTE ORCHESTRATION WITH WIRELESS AND OPTICAL INTEGRATION}

In the TCD Cloud-RAN testbed, the Baseband Units (BBUs) for LTE base stations are implemented using the open-source srsLTE software [7] and centralised into a BBU pool at the OLT (Optical Line Terminal) side of the PON, while the Remote Radio Heads (RRHs) are implemented by Universal Software Radio Peripherals (USRP) at the ONU (Optical Network Unit) side of the PON. The PON is shared between the mobile cells and other broadband traffic, e.g., background traffic, to emulate a scenario were infrastructure is shared between business and residential users.

Figure 1 shows a scenario where the ABNO orchestrator wants to modify the capacity of the converged wirelessoptical system, e.g., following network optimisation operations triggered by measured cell throughput.

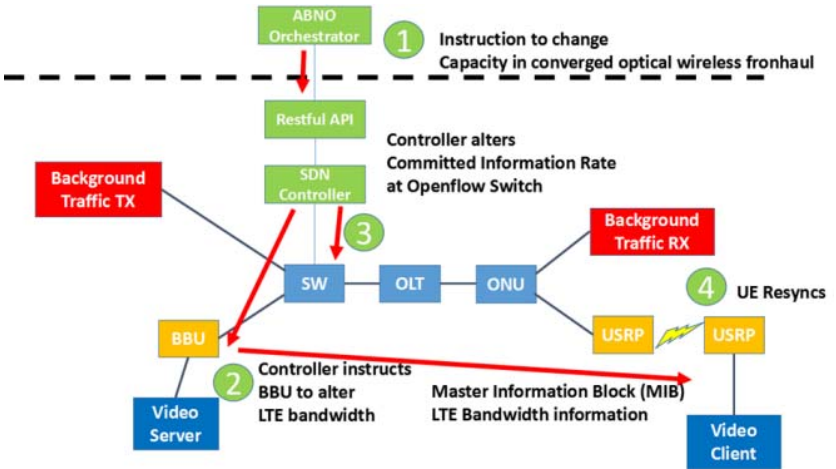

Fig. 1. Event diagram of the orchestrated control on the TCD variable-rate fronthaul testbed.

The ABNO instructs the SDN controller, through the socalled Restful API, to change the bandwidth resource in the converged optical wireless system (Event 1 shown as a green bubble in Fig.1). The SDN controller increases the mobile capacity by sending the BBU an instruction to change the Physical Resource Blocks (PRBs) (Event 2), and simultaneously reconfigures the OpenFlow Switch (indicated as the ' $\mathrm{SW}$ ' blue block) functioning as PON backplane, according to the guaranteed capacity to the fronthaul link (Event 3). After the BBU updates its wireless transmission bandwidth, it broadcasts information about the bandwidth used by the cell through Master Information Block (MIB) messages, as per LTE specifications. The UE listens to this message, and reconfigures accordingly its sampling rate, FFT size, etc., also on the fly (Event 4). The combined downstream flows of I/Q samples and background traffic are fed from the OpenFlow switch to the OLT. The switch separates these links logically via VLAN tags. In the OLT, those VLAN tags are then translated into the XGS-PON addressing format XGEM. At the ONU, the XGEM ports are used to route traffic either to the $10 \mathrm{G}$ Ethernet port connected to the RRU or to the one emulating other PON users (i.e., the background traffic).

Table I reports the relations, experimentally measured in our testbed, between LTE channel bandwidth, fronthaul rate and cell capacity. The additional capacity available over the PON can thus be assigned to other users, a.k.a. the background broadband traffic.

TABLE I. LTE WIRELESS BANDWIDTH TO FRONTHAUL RATE AND APPLICATION RATE MAPPING

\begin{tabular}{rccr}
\hline $\begin{array}{r}\text { Wireless } \\
\text { Bandwidth }\end{array}$ & $\begin{array}{c}\text { PRB } \\
\text { Number }\end{array}$ & $\begin{array}{c}\text { Fronthaul } \\
\text { Rate }\end{array}$ & $\begin{array}{r}\text { Max Cell } \\
\text { Capacity }\end{array}$ \\
\hline $1.4 \mathrm{MHz}$ & 6 & $61 \mathrm{Mbps}$ & $1.8 \mathrm{Mbps}$ \\
$3 \mathrm{MHz}$ & 15 & $121 \mathrm{Mbps}$ & $4.584 \mathrm{Mbps}$ \\
$5 \mathrm{MHz}$ & 25 & $182 \mathrm{Mbps}$ & $7.736 \mathrm{Mbps}$ \\
$10 \mathrm{MHz}$ & 50 & $364 \mathrm{Mbps}$ & $15.264 \mathrm{Mbps}$ \\
$15 \mathrm{MHz}$ & 75 & $485 \mathrm{Mbps}$ & $22.92 \mathrm{Mbps}$ \\
$20 \mathrm{MHz}$ & 100 & $730 \mathrm{Mbps}$ & $30.576 \mathrm{Mbps}$ \\
\hline
\end{tabular}

In the following we detail the steps followed by ABNO to add an E2E flow between a video server located at UNIB and the same video client located at the TCD testbed. The example is meant to show experimenters part of the capabilities of the FUTEBOL facility.

ABNO in time 0 retrieves network resources from the physical topology through the two SDN controllers to build the logical topology, by including information about Virtual Extended Local Area Network (VXLAN) tunnel and TCD SDN switch (SW). Figure 2 introduces the control and data plane integration between the UNIB and the TCD testbeds. Lines in orange represent optical links, lines in blue packet links, and red arrows control and orchestration messages.

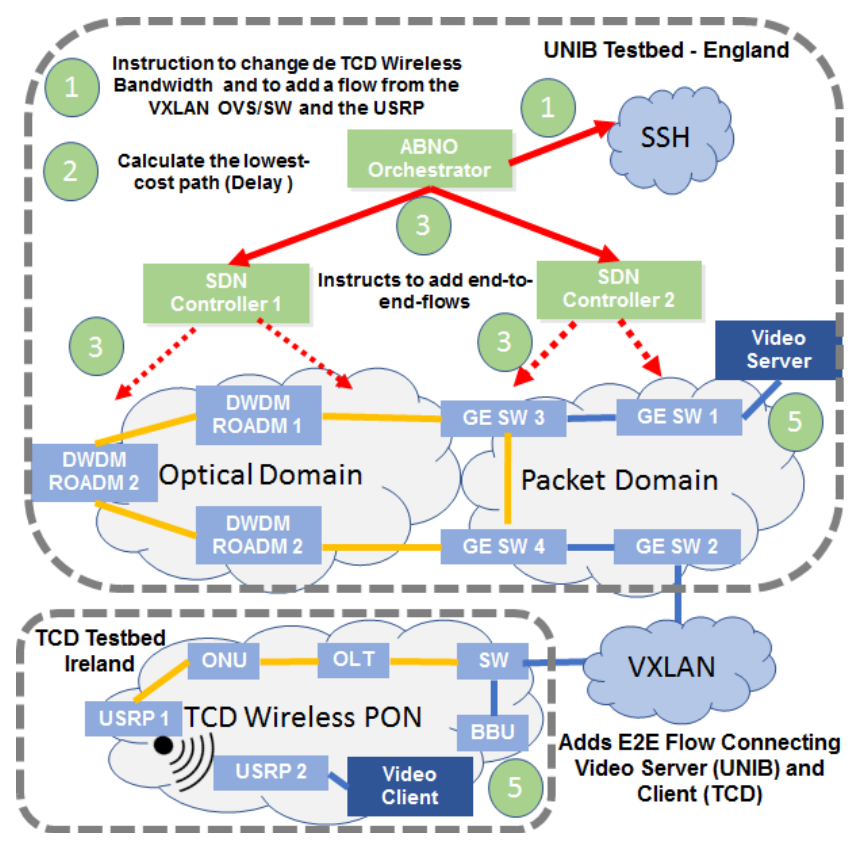

Fig. 2. Integrated control and data plane orchestration between the UNIB Testbed and the TCD Testbed with ABNO.

Step 0: We add the control parameters in the ABNO interface to add a flow between GE SW1 and GE SW2, by connecting UNIB and TCD through VXLAN.

Step 1: ABNO instructs the change in the parameter of the TCD wireless to PRB 100, fronthaul $730 \mathrm{Mbps}$, and 20 
Mhz wireless (Table 1). At the same time ABNO requests the addition of a flow from the SDN switch (SW) connected to the VXLAN and the USRP connecting the video client. The resulted flow will cross the optical link between OLT and ONU to connect the USRP. After receiving the confirmation of the SDN controller from TCD, it continues to Step 2.

Step 2: ABNO calculates the path with the lowest cost (lowest delay in this example) between the SW in TCD and the GE SW 1 connecting the video server.

Step 3: ABNO sends rest-API commands to the SDN Controllers 1 and 2 to add the calculated flow in the DWDM ROADMs and GE SWs.

Step 4: An E2E flow is formed, the bandwidth is allocated in the wireless fronthaul of TCD. The packet, the optical and the wireless domains are now linked.

Step 5: Thevideo streaming begins between server and client.

\section{REAL-TIME ROBOT CONTROL OVER A SW DEFINED WIRELESS-OPTICAL-CLOUD INFRASTRUCTURE}

In this section a different capability of the FUTEBOL facility is shown, by means of an experiment that interested researcher could run from remote.

The main objective of this experiment is to evaluate the impact of convergent SW Defined Wireless-Optical-Edge infrastructure in systems running real-time applications with low E2E latency and high bandwidth requirements. We will demonstrate how communication infrastructure, including optical-wireless integration, and edge datacentre (DC), need to evolve to support future robotics as a service (e.g., for industry 4.0).

This experiment involves three basic groups of resources: i) a so called 'intelligent space', a room equipped with multiple wireless antennas and cameras, connect to ii) an SDN switch, and iii) an optical fiber link, connected to a DC, deployed at the edge of the network, as shown in Fig. 3.

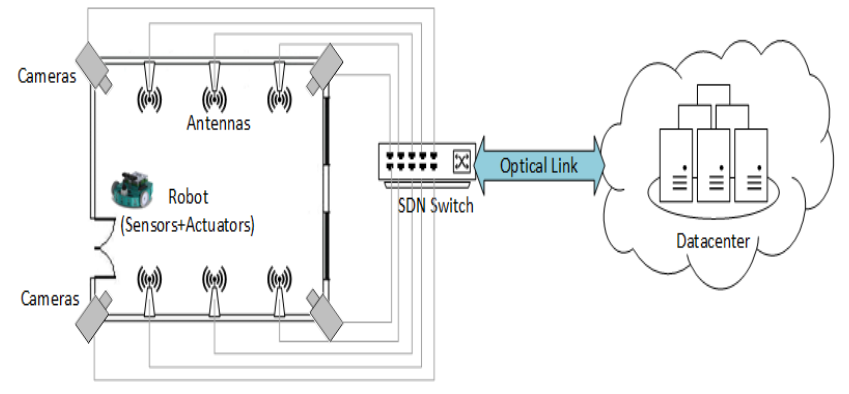

Fig. 3. Real-time robot control over a SW-Defined Wireless-OpticalCloud infrastructure.

The convergent network orchestration is performed by mapping different VLANs as tags to the wireless bands, and the channel in each band, applying SDN OpenFlow matching-action rules for aggregation, isolation and prioritization of wireless network traffic and cameras traffic transported over the optical fiber. Thus, the traffic engineering is conducted by orchestrating multi-domain networks (wireless-packet-optical) in a unified convergent control framework. Moreover, it is also interesting to exploit particularities and asymmetries between upstream and downstream traffic:

a) Upstream traffic from the robot to the edge DC: cameras create a bandwidth hungry traffic. Their individual data streams are dynamically tagged according to the current robot position, when contending for bandwidth in the fiber. Cameras will play different roles in improving the localization precision algorithm depending on their proximity with the robot;

b) Downstream traffic from the edge DC to the robot: this is a light but time-sensitive traffic leaving the DC and (dynamically routed depending on the robot position) reaching the wired infrastructure, connected to the wireless elements. In this case, tagging using VLAN over the fiber enables a sort of in-band signalling. For example, when a VLAN tag, on a given packet on the fiber, reaches out a wireless station, it may impose a band or a wireless channel change for that packet, without going through the (slow) conventional signaling at the control plane.

A more comprehensive description of the experiment and some preliminary results can be found in [8].

\section{CONCLUSION AND FUTURE WORK}

This paper introduces an open facility which is being built under the activities of a research project called FUTEBOL, and that will be made fully available to the research ecosystem at the end of the project. Two exemplary experiments, among the several ones that could be run, are described, stressing the importance of merging wireless and optical networks. Next steps will involve the enhancements of the set of testbeds composing the FUTEBOL facility, and the fulfilment of the project objectives, as described in [6].

\section{ACKNOWLEDGMENT}

This work has received funding from the European Union Horizon 2020 programme for research, technological development, and demonstration under grant agreement no. 688941 (FUTEBOL), as well from the Brazilian Ministry of Science, Technology and Innovation (MCTI) through RNP and CTIC.

\section{REFERENCES}

[1] Availabe online: https://www.gartner.com/newsroom/id/3598917.

[2] B. Raaf, M. Faerber, B. Badic, and V. Frascolla, "Key technology advancements driving mobile communications from generation to generation," Intel Technology Journal, vol. 18, issue 1, 2014.

[3] Available online: https://ec.europa.eu/digital-single-market/en/futureinternet-research-and-experimentation.

[4] FUTEBOL website. Available online: www.ict-futebol.org.br.

[5] C. Dominicini et al., "Enabling experimental research through converged orchestration of optical, wireless, and cloud domains," in $27^{\text {th }}$ European Conference on Networks and Communications (EuCNC), 2018

[6] P. Marques, et. al., "Experiments Overview of the EU-Brazil FUTEBOL Project" in $26^{\text {th }}$ European Conference on Networks and Communications (EuCNC), 2017.

[7] Available online: https://softwareradiosystems.com.

[8] V. M. G. Martinez, et. al., "Ultra reliable communication for robot mobility enabled by sdn splitting of wifi functions," in IEEE Symposium on Computers and Communications (ISCC), 2018. 